\title{
5-dimensional Quantum Gravity Effects in Exclusive Double Diffractive Events
}

\author{
A.V. Kisselev*, V.A. Petrov ${ }^{\dagger}$ and R.A. Ryutin ${ }^{\ddagger}$ \\ Institute for High Energy Physics, 142281 Protvino, Russia
}

\begin{abstract}
The experimentally measurable effects related to extra dimensional gravity in a RS-type brane world are estimated. Two options of the RS framework (namely, with small and large curvature) are considered. It is shown that both can be detected by the joint experiment of the CMS and TOTEM Collaborations at the LHC.
\end{abstract}

\section{Massive Gravitons and Radion in the RS model}

There is no doubt that the discovery of particles like Higgs boson is the fundamental goal, but it will not solve a very important problem of the hierarchy between the electro-weak $(246 \mathrm{GeV})$ and Planck $\left(2.4 \cdot 10^{18} \mathrm{GeV}\right)$ scales. Some models have been proposed recently, which resolve the problem without supersymmetry but rather with help of space-time with extra dimensions. For instance, so-called ADD model [1] "explains" the large value of the Planck scale by the large size of compact extra dimensions. Such theories open way for many new experimental studies.

In particular, the model of Randall and Sundrum (RS) [2, 3] seems to be the most economic introducing only one extra dimension which has not to be large. It is based on an exact solution for gravity in a five-dimensional space-time, where the extra spatial dimension is a "folded" circle. Let $\left\{z^{M}\right\}=\left\{\left(x^{\mu}, y\right)\right\}, M=0,1,2,3,4$, be its coordinates. Namely, $y$ is the coordinate along the fifth dimension, while $\left\{x^{\mu}\right\}, \mu=0,1,2,3$, are the coordinate in a four-dimensional space-time. The background metric of the model is of the form (contribution of the matter energy-momentum tensor is neglected):

$$
d s^{2}=\gamma_{M N}(z) d z^{M} d z^{N}=e^{2 \kappa|y|} \eta_{\mu \nu} d x^{\mu} d x^{\nu}+d y^{2} .
$$

Here $y=r_{c} \theta(-\pi \leqslant \theta \leqslant \pi), r_{c}$ being the "radius" of the extra dimension, and the parameter $\kappa$ defines the scalar curvature of the five-dimensional space-time. The points $\left(x^{\mu}, y\right)$ and $\left(x^{\mu},-y\right)$ are identified, and the periodicity condition, $\left(x^{\mu}, y\right)=\left(x_{\mu}, y+2 \pi r_{c}\right)$, is imposed. In Eq. (II) $\eta_{\mu \nu}$ is the Minkowski metric.

\footnotetext{
${ }^{*}$ E-mail address: alexandre.kisselev@mail.ihep.ru

${ }^{\dagger}$ E-mail address: vladimir.petrov@mail.ihep.ru

${ }^{\ddagger}$ E-mail address: ryutin@th1.ihep.su
} 
We consider the so-called RS1 model [2] which has two 4-dimensional branes with equal and opposite tension located at the point $y=0$ (called the TeV brane, or visible brane) and at $y=\pi r_{c}$ (referred to as the Plank brane). All SM fields are constrained to the $\mathrm{TeV}$ brane, while the gravity propagates in all five dimensions (bulk).

Since the warp factor $e^{2 \kappa|y|}$ is equal to 1 on the TeV brane, four-dimensional coordinates $\left\{x^{\mu}\right\}$ are Galilean and we have a correct determination of the graviton fields on this brane. For a zero mode sector of the effective theory, one obtains a relation between the (reduced) Planck mass and the (reduced) fundamental gravity scale in five dimensions $\bar{M}_{5}$ :

$$
\bar{M}_{P l}^{2}=\frac{\bar{M}_{5}^{3}}{\kappa}\left(e^{2 \pi \kappa r_{c}}-1\right) .
$$

In the linear approximation, one can parametrize the metric $g_{M N}$ as

$$
g_{M N}(z)=\gamma_{M N}(z)+\frac{2}{\bar{M}_{5}^{3 / 2}} h_{M N}(z)
$$

The invariance of the gravitational action under general coordinate transformations means that the Lagrangian is invariant under gauge transformations of the field $h_{M N}(z)$ (for details, see Refs. [4, 5]). If we impose so-called unitary gauge [5], we get:

$$
h_{\mu 4}(x, y)=0, \quad h_{44}(x, y)=\phi(x),
$$

where $\phi(x)$ is a massless scalar field which depends on four-dimensional coordinates only. This new degree of freedom, called radion, corresponds to distance oscillations between the branes.

This massless scalar field would lead to such a change of the usual gravitational interaction on the visible brane which is totally excluded by experimental data. However, if the radion acquires the mass of the order of $100 \mathrm{GeV}$ [6], this will not contradict the experimental data, i.e. the radion could be the lightest massive scalar excitation of the RS model.

The field $h_{\mu \nu}(x, y)$ (with a radion contribution singled out) is decomposed into a massless mode $h_{\mu \nu}^{(0)}(x)$ ("classical" graviton) and "Kaluza-Klein" (KK) modes $h_{\mu \nu}^{(n)}(x)$ which describe massive gravitons. The mass spectrum of the KK gravitons on the TeV brane is the following:

$$
m_{n}=x_{n} \kappa, \quad n=1,2 \ldots,
$$

where $x_{n}$ are zeros of the Bessel function $J_{1}(x)$, with $x_{n} \simeq \pi n$ at large $\mathrm{n}$. The interaction Lagrangian on the visible $(\mathrm{TeV})$ brane looks like

$$
\mathcal{L}_{i n t}=-\frac{1}{\bar{M}_{P l}} T^{\mu \nu} h_{\mu \nu}^{(0)}-\frac{1}{\Lambda_{\pi}} T^{\mu \nu} \sum_{n=1}^{\infty} h_{\mu \nu}^{(n)}+\frac{1}{\sqrt{3} \Lambda_{\pi}} T_{\mu}^{\mu} \phi
$$

Here $T^{\mu \nu}$ is the energy-momentum tensor of the matter on the brane, $h_{\mu \nu}^{(n)}$ is the graviton field with the KK-number $n$ and mass $m_{n}$ (5) . The parameter

$$
\Lambda_{\pi}=\left(\frac{\bar{M}_{5}^{3}}{\kappa}\right)^{1 / 2}
$$


is a physical scale on the TeV brane. As one can see from (6), the radion field is coupled to the trace of the energy-momentum tensor.

Let us consider two possibilities to satisfy relation (2). One possibility (we will call it "large curvature option") is to put

$$
\kappa \simeq \bar{M}_{5} \sim 1 \mathrm{TeV}
$$

that corresponds to $\kappa r_{c}=11.3$ in Eq. (2). There is a series of KK massive graviton resonances, with the lightest one having a mass of order $1 \mathrm{TeV}$. As for the radion, it is coupled rather strongly to the SM fields (mainly, to gluons) since $\Lambda_{\pi} \sim 1 \mathrm{TeV}$.

Another possibility (we will call it "small curvature option" [7, 8]) is to take

$$
\kappa \ll \bar{M}_{5} \sim 1 \mathrm{TeV}
$$

In such a case, the mass splitting $\Delta m \simeq \pi \kappa$ can be chosen smaller than the energy resolution of LHC experiments. For instance, for $\kappa r_{c}=9.7$, we get $\pi \kappa=50 \mathrm{MeV}$, and the mass of the lightest $\mathrm{KK}$ excitation is $m_{1}=60.5 \mathrm{MeV}$. This case is not favorable for the production of the radion, since the coupling is defined by $\Lambda_{\pi}=\left(\bar{M}_{5} / 1 \mathrm{TeV}\right)^{3 / 2} 140 \mathrm{TeV}$ and is two orders of magnitude smaller than in the previous case.

\section{Mechanism of the Exclusive Double Diffraction}

The main goal of our paper is to analyze experimental possibilities to detect effects of the extra dimensions in exclusive double diffractive event (EDDE):

$$
p+p \rightarrow p+\text { "gap" }+X+\text { "gap" }+p,
$$

where $X$ represents a particle strongly coupled to two-gluon states (say, the radion, SM Higgs, KK graviton, meson containing heavy quarks or glueball).

Unique advantages of this process are well-known (see [9]-13] and references therein): a) clear signature of the process; b) possibility to use the "missing mass method", that improves the mass resolution; c) background is strongly suppressed; d) spin-parity analysis of the central system can be done. All these properties can be realized in common CMS/TOTEM detector measurements at LHC [14.

The exclusive double diffractive process is related to the dominant amplitude of the exclusive two-gluon production. Driving mechanism of this processes is the Pomeron.

To calculate an amplitude of the process (10), we use an approach which was considered in detail in Ref. 9]. In the framework of this approach, the amplitude can be sketched as shown in Fig. 1. After the tensor contraction of the amplitudes $T_{1,2}$ with the gluon-gluon fusion vertex, the full "bare" amplitude $T_{X}$ depicted in Fig. 19 looks like

$$
T_{X}=\frac{2}{\pi} c_{g p}^{2} e^{b\left(t_{1}+t_{2}\right)}\left(-\frac{s}{M_{X}^{2}}\right)^{\alpha_{P}(0)} F_{g g \rightarrow X} I_{s} .
$$

Here

$$
\begin{aligned}
b & =\alpha_{P}^{\prime}(0) \ln \left(\frac{\sqrt{s}}{M_{X}}\right)+b_{0}, \\
b_{0} & =\frac{1}{4}\left(\frac{r_{p p}^{2}}{2}+r_{g p}^{2}\right),
\end{aligned}
$$


a)

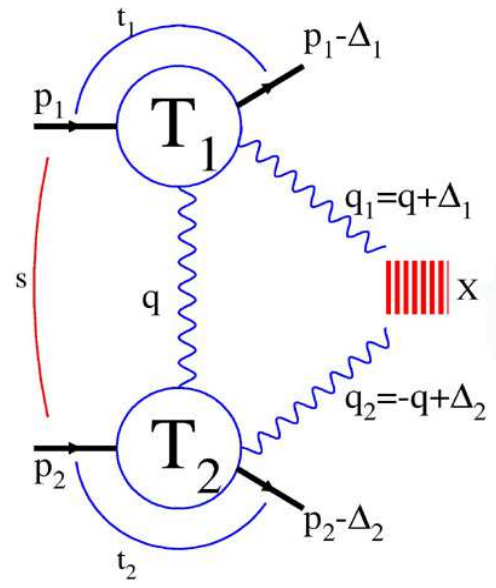

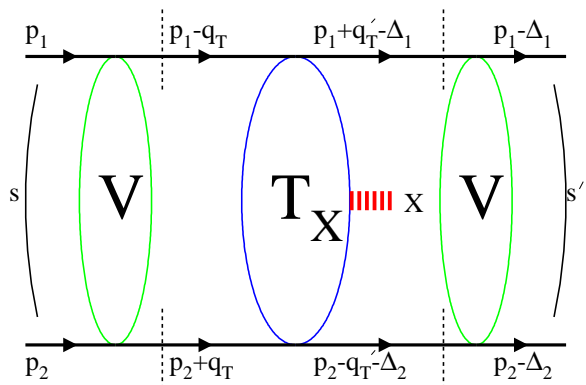

b)

Figure 1: Model for EDDE: a) amplitude of the exclusive double diffractive production $T_{X}$ without unitary corrections; b) amplitude $T_{X}^{\text {unit }}$ with accounting for "soft" re-scattering corrections $V$ in the initial and final states.

with the parameters of the "hard" Pomeron trajectory, that appears to be the most relevant in our case, presented in Table 1. The last factor in the RHS of Eq. (11) is

$$
I_{s}=\int_{0}^{M_{X}^{2} / 4} \frac{d l^{2}}{l^{4}} F_{s}\left(l^{2}\right)\left(\frac{l^{2}}{s_{0}+l^{2} / 2}\right)^{2 \alpha_{P}(0)}
$$

where $l^{2}=-q^{2} \simeq \mathbf{q}^{2}$, and $s_{0}$ is a scale parameter of the model which is also used in the global fitting of the data on $p p(p \bar{p})$ scattering for on-shell amplitudes [15]. The fit gives $s_{0} \simeq 1 \mathrm{GeV}^{2}$. If we take into account the emission of virtual "soft" gluons, while prohibiting the real ones, that could fill rapidity gaps, it results in a Sudakov-like suppression [16]:

$$
F_{s}\left(l^{2}\right)=\exp \left[-\frac{3}{2 \pi} \int_{l^{2}}^{M_{X}{ }^{2} / 4} \frac{d p_{T}^{2}}{p_{T}^{2}} \alpha_{s}\left(p_{T}^{2}\right) \ln \left(\frac{M_{X}^{2}}{4 p_{T}^{2}}\right)\right],
$$

The off-shell gluon-proton amplitudes $T_{1,2}$ are obtained in the extended unitary approach [17]. The "hard" part of the EDDE amplitude, $F_{g g \rightarrow X}$, is the usual gluon-gluon fusion amplitude calculated perturbatively in the SM or in its extensions.

The data on total cross-sections demand unambiguously the Pomeron with larger-thanone intercept, thereof the need in unitarization. The amplitude with unitary corrections, $T_{X}^{u n i t}$, are depicted in Fig. 1b. It is given by the following analytical expressions:

$$
\begin{aligned}
T_{X}^{u n i t}\left(p_{1}, p_{2}, \Delta_{1}, \Delta_{2}\right) & =\frac{1}{16 s s^{\prime}} \int \frac{d^{2} \mathbf{q}_{T}}{(2 \pi)^{2}} \frac{d^{2} \mathbf{q}_{T}^{\prime}}{(2 \pi)^{2}} V\left(s, \mathbf{q}_{T}\right) \\
& \times T_{X}\left(p_{1}-q_{T}, p_{2}+q_{T}, \Delta_{1 T}, \Delta_{2 T}\right) V\left(s^{\prime}, \mathbf{q}_{T}^{\prime}\right) \\
V\left(s, \mathbf{q}_{T}\right) & =4 s(2 \pi)^{2} \delta^{2}\left(\mathbf{q}_{T}\right)+4 s \int d^{2} \mathbf{b} e^{i \mathbf{q}_{T} \mathbf{b}}\left[e^{i \delta_{p p \rightarrow p p}}-1\right]
\end{aligned}
$$


Table 1: Phenomenological parameters of the "hard" Pomeron trajectory obtained from the fitting of the HERA and Tevatron data (see [9], [18]), and data on $p p(p \bar{p})$ scattering [15].

\begin{tabular}{||c|c|c|c|c||}
\hline$\alpha_{P}(0)$ & $\alpha_{P}^{\prime}(0), \mathrm{GeV}^{-2}$ & $r_{p p}^{2}, \mathrm{GeV}^{-2}$ & $r_{g p}^{2}, \mathrm{GeV}^{-2}$ & $c_{g p}$ \\
\hline 1.203 & 0.094 & 2.477 & 2.54 & 3.3 \\
\hline
\end{tabular}

where $\Delta_{1 T}=\Delta_{1}-q_{T}-q_{T}^{\prime}, \Delta_{2 T}=\Delta_{2}+q_{T}+q_{T}^{\prime}$, and the eikonal function $\delta_{p p \rightarrow p p}$ can be found in Ref. [15]. Left and right parts of the diagram in Fig. 10 denoted by $V$ represent "soft" re-scattering effects in initial and final states, i.e. multi-Pomeron exchanges. As was shown in [11, these "outer" unitary corrections strongly reduce the value of the corresponding cross-section and change an azimuthal angle dependence.

\section{Exclusive Particle Production in Double Diffrac- tive Processes}

\subsection{Radion Production in EDDE}

In this subsection, we will study the radion production in EDDE in the case of the "large curvature option" (8), taking into account an effect of a mixing between the radion and SM Higgs. ${ }^{1}$

Since the radion has the same quantum numbers as the standard Higgs boson $h$, there could be the mixing between them which leads to significant changes in the cross-section of hadroproduction of both [20]. This problem and its phenomenological consequences were studied by many authors (see, for example, [21]).

The following Lagrangian describes the Higgs-radion interaction:

$$
\mathcal{L}_{h-\phi}=-\frac{6 \xi v}{\Lambda_{\phi}} \phi \square h
$$

Here $v=246 \mathrm{GeV}$ and $\Lambda_{\phi}=\sqrt{3} \Lambda_{\pi}$ are the vacuum expectation values of the Higgs and radion fields, respectively. The quantity $\xi$ is the Higgs-radion mixing parameter. For $\xi=0$, the radion decouples from the Higgs. If $\xi \neq 0$, the radion and the SM Higgs boson mix into the two new eigenstates. Branching fractions for transitions into SM states can be quite different depending on $\xi$ and $\Lambda_{\phi}$.

The results of our calculations of the total cross sections are presented in Fig. 2a and $2 \mathrm{~b}$ for various values of the mixing parameter $\xi$ and $\Lambda_{\phi}$. As we see from these figures, due to the Higgs-radion mixing, the cross-sections for a single radion production in EDDE can be larger than those for the SM Higgs boson. Estimated number of events per year for the integrated luminosity $30 \mathrm{fb}^{-1}$ and registration efficiency $10 \%$ are given in Table 2 .

\footnotetext{
${ }^{1} \mathrm{~A}$ similar problem for an inclusive production was considered in [19].
} 


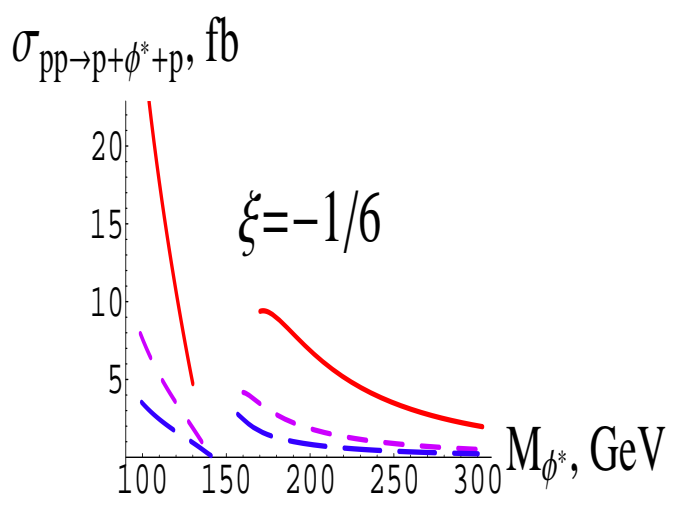

a)

Figure 2: The cross section for the production of the radion in EDDE vs. mass of the observable eigenstate $\phi^{*}$. Mass parameter of the Higgs is $M(h)=150 \mathrm{GeV}$. Three curves correspond (from top to bottom) to $\Lambda_{\phi}=1 \mathrm{TeV}, 2 \mathrm{TeV}, 4 \mathrm{TeV}$. a) $\xi=-1 / 6$; b) $\xi=1 / 6$.

Table 2: The expected number of EDDE's for the production of the radion for various values of $\Lambda_{\phi}$, and $\xi=1 / 6(-1 / 6)$. Higgs mass parameter $M(h)$ is set to $150 \mathrm{GeV}$. A realistic value of the total efficiency of an event registration is assumed to be $10 \%$ (as it was estimated from fast Monte-Carlo simulation for the SM Higgs case), and integrated luminosity is taken to be $30 \mathrm{fb}^{-1}$.

\begin{tabular}{||c||c|c|c||}
\hline \multicolumn{1}{||c||}{} & \multicolumn{3}{c||}{$M_{\phi^{*}}, \mathrm{GeV}$} \\
\hline$\Lambda_{\phi}, \mathrm{TeV}$ & 100 & 120 & 130 \\
\hline 1 & $116(78)$ & $64(31)$ & $48(13)$ \\
\hline 2 & $31(22)$ & $20(10)$ & $17(4)$ \\
\hline 4 & $8(5)$ & $6(3)$ & $5(1)$ \\
\hline
\end{tabular}

In Figs. 3 and 4 one can see $\sigma\left(\phi^{*}\right) \cdot B r$ for various decay modes. The values of the parameters are $\xi=1 / 6,-1 / 6$ and $\Lambda_{\phi}=2 \mathrm{TeV}, M(h)=150 \mathrm{GeV}$. A significance of the event is estimated to be greater than $3 \sigma$ in the region of $M_{\phi^{*}}<140 \mathrm{GeV}$ (bj decay mode) and $\Lambda_{\phi}<5 \mathrm{TeV}$. For larger radion masses $\left(Z Z\right.$ and $W^{+} W^{-}$decay modes), we get a similar significance for the integrated luminosity $100 \mathrm{fb}^{-1}$.

\subsection{Production of the KK Gravitons in EDDE}

As was shown in Ref. [8, the "small curvature option" of the RS model is similar to the ADD model of gravity in a flat space-time with one compact extra dimension [1] up to the following formal replacement in the KK sector:

$$
\bar{M}_{P l} \rightarrow \Lambda_{\pi}, \quad R_{c} \rightarrow \frac{1}{\pi \kappa}
$$



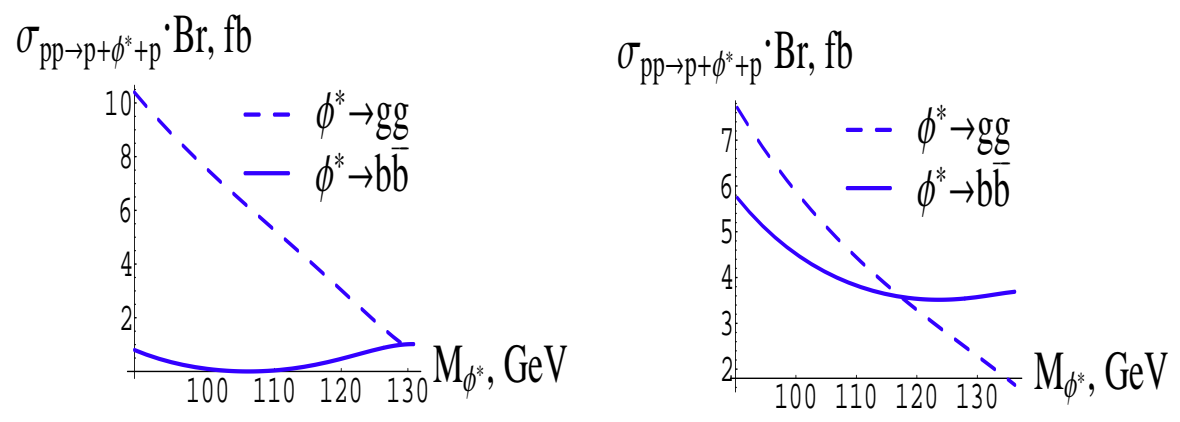

a)

b)

Figure 3: The cross section for the production of the radion in EDDE multiplied by the branching fraction vs. mass of the observable eigenstate $\phi^{*}$. The curves correspond to $g g$ decay mode (dash one) and $b \bar{b}$ (solid one). The parameters of the model are: a) $\Lambda_{\phi}=$ $2 \mathrm{TeV}, M(h)=150 \mathrm{GeV}, \xi=-1 / 6$; b) $\Lambda_{\phi}=2 \mathrm{TeV}, M(h)=150 \mathrm{GeV}, \xi=1 / 6$.
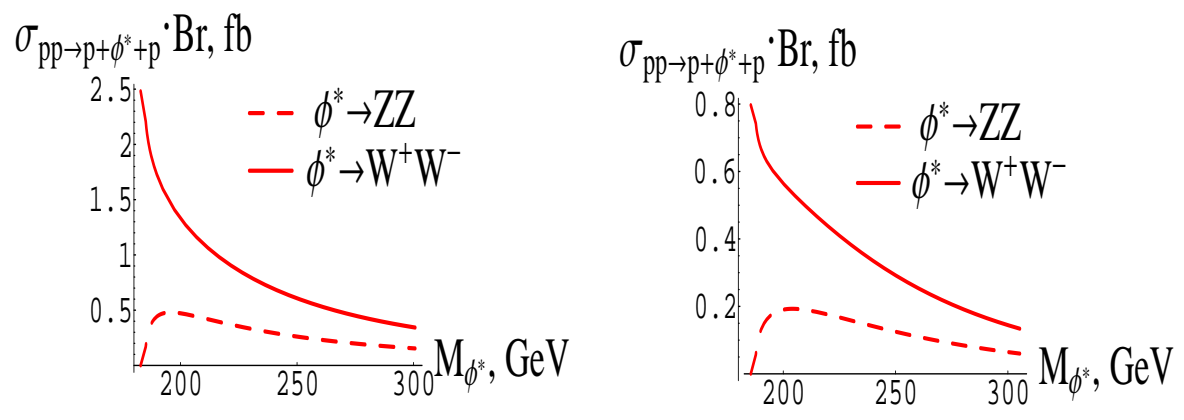

a)

b)

Figure 4: The cross section for the production of the radion in EDDE multiplied by the branching fraction vs. mass of the observable eigenstate $\phi^{*}$. The curves correspond to $Z Z$ decay mode (dash one) and $W^{+} W^{-}$(solid one). The parameters of the model are: a) $\Lambda_{\phi}=2 \mathrm{TeV}, M(h)=150 \mathrm{GeV}, \xi=-1 / 6$; b) $\Lambda_{\phi}=2 \mathrm{TeV}, M(h)=150 \mathrm{GeV}, \xi=1 / 6$.

Here $R_{c}$ is a radius of the extra dimension in the flat space-time.

As was already mention in the end of Section 11 this case is not favorable for the production of the radion, due to a large value of the scale $\Lambda_{\pi}$ (remember that it defines the coupling of the radion to the SM fields, see the last term in Eq. (6) ). On the contrary, KK graviton production cross section does not depend on $\Lambda_{\pi}$, but it is defined by the 5-dimensional Planck scale $\bar{M}_{5}$ only, as it will be demonstrated below.

In the case of a small value of the curvature parameter $\kappa$ (9), we have the spectrum of the KK gravitons with the small mass splitting. Since the widths of the massive gravitons, $\Gamma_{n}$, are very small [22,

$$
\frac{\Gamma_{n}}{m_{n}} \simeq\left(0.31 \frac{m_{n}}{\Lambda_{\pi}}\right)^{2},
$$

the KK gravitons behave like extremely narrow massive spin-2 resonances. Thus, a typical collider signature of the KK graviton production is an imbalance in missing mass of final states with a continuous mass distribution, which could be 


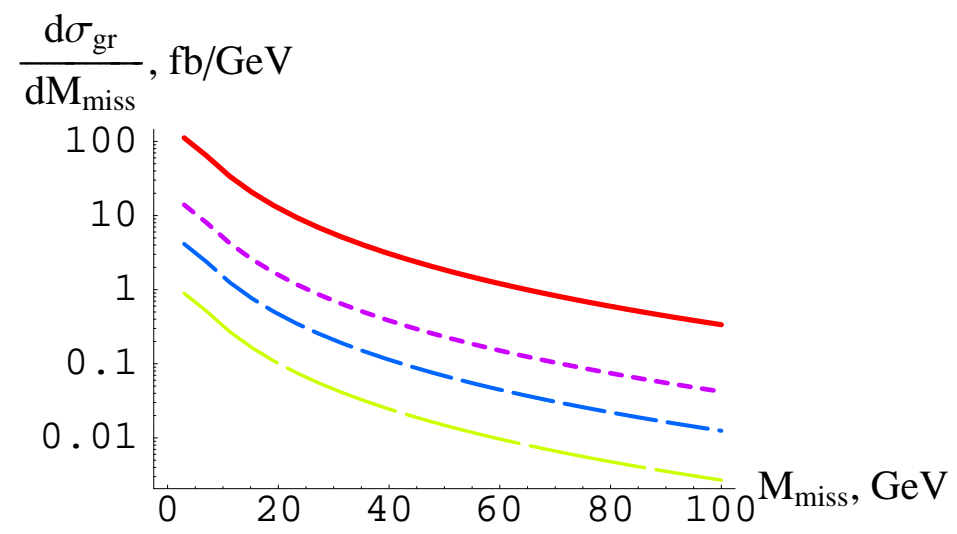

Figure 5: The distribution in the missing mass in the double diffractive production of the KK gravitons in the RS model with the small curvature. The curves correspond (from top to bottom) to $\bar{M}_{5}=1 \mathrm{TeV}, 2 \mathrm{TeV}, 3 \mathrm{TeV}$, and $5 \mathrm{TeV}$.

observed in the EDDE-like process of the type

$$
p+p \rightarrow p+\text { "nothing" }+p .
$$

In other words, one should look for a double diffractive process with a missing mass $M_{\text {miss }}$ and "nothing else" in the central region.

It can be shown that the distribution in $M_{m i s s}$ is proportional to

$$
\frac{d \sigma_{g r}}{d M_{\text {miss }}} \sim \frac{1}{\kappa \Lambda_{\pi}^{2}} \sim \frac{1}{\bar{M}_{5}^{3}}
$$

In other words, both the distribution in $M_{\text {miss }}$ and total cross section $\sigma_{g r}$ are defined by the fundamental Planck scale in five warped dimensions only, not by the values of $\kappa$ and $\Lambda_{\pi}$ separately. Since $\bar{M}_{5} \sim 1 \mathrm{TeV}$, we expect that corresponding cross sections will be large enough to be measured in the common experiment of the CMS and TOTEM Collaborations [14].

To estimate $d \sigma_{g r} / d M_{\text {miss }}$ and the total cross section of the graviton production in EDDE, $\sigma_{g r}$, numerically we use the model of EDDE described in Section 2 . The results of our calculations are presented in Figs [5, 6] and Table 4. The missing mass distribution $d \sigma_{g r} / d M_{\text {miss }}$ is shown in Fig. [5. In Fig. 6] one can see $\sigma_{g r}$ as a function of $\bar{M}_{5}$. The curves in Fig. 6] correspond to various values of $M_{0}$, a lower cut on $M_{\text {miss }}$, which is imposed to suppress the soft photon/graviton contributions, and to provide applicability of our mechanism of exclusive double diffractive production. The cut of $M_{0}$ is chosen to be 3,14 and $30 \mathrm{GeV}$. An upper cut on $M_{\text {miss }}$ is taken to be $90 \mathrm{GeV}$ in order to suppress a possible background from the process $p+p \rightarrow p+$ neutrinos $+p$. Note that this upper cut-off does not significantly reduce a signal due to a rapid fall-off of $d \sigma_{g r} / d M_{m i s s}$ in variable $M_{\text {miss }}$ (see Fig. [5).

Let us underline that the results obtained in this Section do not depend on the physical scale $\Lambda_{\pi}$ (or curvature parameter $\kappa$ ), but depend only on the fundamental gravity scale in five dimensions $\bar{M}_{5}$. 


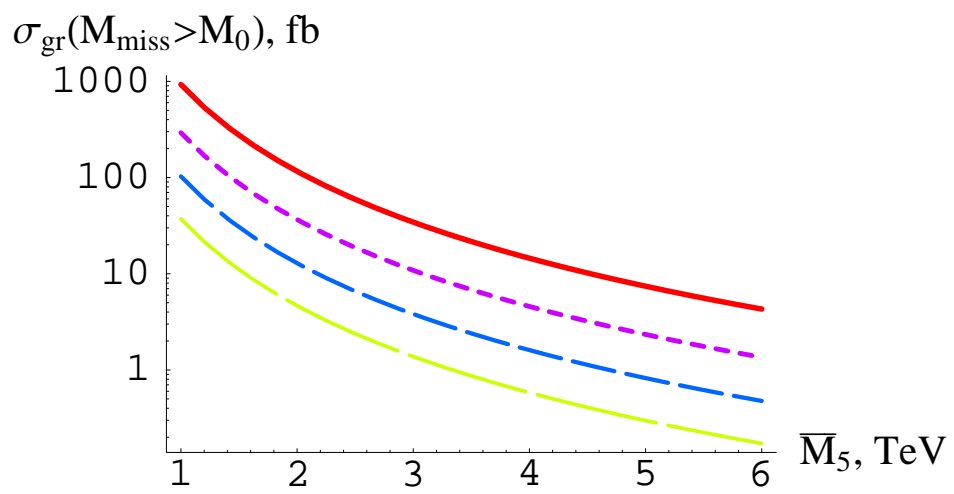

Figure 6: The cross section for double diffractive production of the KK gravitons with masses larger that $M_{0}$ as a function of 5-dimensional Planck scale $\bar{M}_{5}$. The curves correspond (from top to bottom) to $M_{0}=3 \mathrm{GeV}, 14 \mathrm{GeV}, 30 \mathrm{GeV}$, and $50 \mathrm{GeV}$.

Table 3: The integrated luminosity related to $M_{0}$, possible lower experimental limit on missing mass measurements [14. Here the total efficiency is assumed to be $100 \%$. To get more realistic estimation, one needs further Monte-Carlo simulations.

\begin{tabular}{||c||c|c|c|c||}
\hline$M_{0}, \mathrm{GeV}$ & 3 & 14 & 30 & 50 \\
\hline $\mathcal{L}, \mathrm{fb}^{-1}$ & 0.3 & $0.3-30$ & $30-300$ & $30-300$ \\
\hline
\end{tabular}

Table 4: The expected number of EDDE's for the production of the KK gravitons for various values of the fundamental gravity scale $\bar{M}_{5}$ and parameter $M_{0}$. The integrated luminosity is taken from Table 3 .

\begin{tabular}{||c||c|c|c|c||}
\hline \multicolumn{1}{||c||}{} & \multicolumn{3}{c||}{$M_{0}, \mathrm{GeV}$} \\
\hline $\bar{M}_{5}, \mathrm{TeV}$ & 3 & 14 & 30 & 50 \\
\hline 1 & 280 & $87-8.7 \cdot 10^{3}$ & $3.0 \cdot 10^{3}-3.0 \cdot 10^{4}$ & $1.11 \cdot 10^{3}-1.11 \cdot 10^{4}$ \\
\hline 2 & 36 & $11-1.1 \cdot 10^{2}$ & $390-3.9 \cdot 10^{3}$ & $138-1.38 \cdot 10^{3}$ \\
\hline 3 & 9 & $3-300$ & $114-1.14 \cdot 10^{3}$ & $42-420$ \\
\hline 5 & 3 & $0.7-70$ & $24-240$ & $9-90$ \\
\hline
\end{tabular}

\section{Conclusions}

In this paper we present the evaluation of experimentally observable signals due to extra dimensional gravity effects in a RS-type brane world. 
Our estimates show that both extreme options of the RS-type scenario (i.e. those of small and large curvature) give distinctive signals which could be fairly detected at the LHC (possible joint measurements by the CMS and TOTEM LHC Collaborations).

\section{Acknowledgements}

This work is supported by grants PICS2910 of the CNRS and RFBR-04-02-17299.

\section{References}

[1] N. Arkani-Hamed, S. Dimopoulos and G. Dvali, Phys. Lett. B 429 (1998) 263;

I. Antoniadis, N. Arkani-Hamed, S. Dimopoulos and G. Dvali, Phys. Lett. B 436 (1998) 257;

N. Arkani-Hamed, S. Dimopoulos and G. Dvali, Phys. Rev. D 59 (1999) 086004.

[2] L. Randall and R. Sundrum, Phys. Rev. Lett. 83 (1999) 3370.

[3] L. Randall and R. Sundrum, Phys. Rev. Lett. 83 (1999) 4690; J. Lykken and L. Randall, JHEP 06 (2000) 014.

[4] C. Charmousis, R. Gregory and V.A. Rubakov, Phys. Rev. D 62 (2000) 067505;

I.Ya. Aref'eva, M.G. Ivanov, W.Mück, K.S. Viswanathan and I.V. Volovich, Nucl. Phys. B 590 (2000) 273.

[5] E.E. Boos, Yu.A. Kubyshin, M.N. Smolyakov and I.P. Volobuev, Class. Quant. Grav. 19 (2002) 4591.

[6] W.D. Goldberger and M.B. Wise, Phys.Rev. Lett. 83 (1999) 4922;

C. Csáki et al., Phys. Rev. D 62 (1999) 045015;

C. Csáki, M. Graesser and G.D. Kribs, ibid D 63 (2001) 064020.

[7] G.F. Giudice, T. Plehn and A. Strumia, Nucl. Phys. B 706 (2005) 455.

[8] A.V. Kisselev and V.A. Petrov, arXiv:hep-ph/0504203, to appear in Phys. Rev. D.

[9] V.A. Petrov and R.A. Ryutin, JHEP 0408 (2004) 013.

[10] V.A. Petrov and R.A. Ryutin, Eur. Phys. J. C 36 (2004) 509.

[11] V.A. Petrov, R.A. Ryutin, A.E. Sobol and J.-P. Guillaud, arXiv:hep-ph/0409118, to appear in JHEP.

[12] V.A. Khoze, A.D. Martin, M.G. Ryskin, Eur. Phys. J. C 19 (2001) 477;

A. De Roeck, V.A. Khoze, A.D. Martin, R. Orava, M.G. Ryskin, Eur. Phys. J. C 25 (2002) 391;

V.A. Khoze, A.D. Martin, M.G. Ryskin, Eur. Phys. J. C 23 (2002) 311;

A.D. Martin, M.G. Ryskin and V.A. Khoze, Phys. Rev. D 56 (1997) 5867. 
[13] A.B. Kaidalov, V.A. Khoze, A.D. Martin and M.G. Ryskin, Eur. Phys. J. C 31 (2003) 387 ;

V.A. Khoze, A.D. Martin and M.G. Ryskin, Eur. Phys. J. C 24 (2002) 581.

[14] K. Eggert, M. Oriunno and M. Bozzo, TOTEM Technical Design Report, CERNLHCC-2004-002;

M. Deile, Talk given at the Workshop "Physics at LHC" (July 2004, Vienna), arXive:hep-ex/0503042.

[15] V.A. Petrov and A.V. Prokudin, Eur. Phys. J. C 23 (2002) 135.

[16] V.A. Khoze, A.D. Martin and M.G. Ryskin, Eur. Phys. J. C 14 (2000) 525; ibid. C 21 (2001) 99;

V.A. Khoze, arXive:hep-ph/0105224

[17] V.A. Petrov, Talk given at the 6th Workshop on Elastic and Diffractive Scattering (20-24 Jun 1995, Blois, France). In Blois 1995, Frontiers in strong interactions, p. $139-143$.

[18] V.A. Petrov, A.V. Prokudin and R.A. Ryutin, Czech. J. Phys. 55 (2005) 17.

[19] M. Battaglia, S. De Curtis, A. De Roeck, P. Dominici, and J.F. Gunion, Phys. Lett. B 568 (2003) 92 .

[20] G.F. Giudice, R. Rattazzi and J.D. Wells, Nucl. Phys. B 595 (2001) 250.

[21] M. Chaichian, A. Datta, K. Huitu and Z. Yu, Phys. Lett. B 524 (2002) 161.

[22] A.V. Kisselev, arXive:hep-ph/0412367, to appear in Eur. Phys. J. C. 